OPEN ACCESS

Edited by:

Feng Liu,

Tianjin Medical University General Hospital, China

Reviewed by:

Zaixu Cui,

University of Pennsylvania,

United States

Qinghua He,

Southwest University, China

*Correspondence:

Wei Qin

wqin@xidian.edu.cn

${ }^{\dagger}$ These authors have contributed equally to this work

Specialty section: This article was submitted to Brain Imaging Methods, a section of the journal Frontiers in Neuroscience

Received: 13 December 2018 Accepted: 18 April 2019

Published: 08 May 2019

Citation:

$X u Z$, Yang $X$, Gao $M$, Liu L, Sun J, Liu P and Qin W (2019) Abnormal Resting-State Functional Connectivity in the Whole Brain in Lifelong Premature Ejaculation Patients Based on Machine Learning Approach. Front. Neurosci. 13:448. doi: 10.3389/fnins.2019.00448

\section{Abnormal Resting-State Functional Connectivity in the Whole Brain in Lifelong Premature Ejaculation Patients Based on Machine Learning Approach}

\author{
Ziliang Xu' ${ }^{1+}$, Xuejuan Yang ${ }^{1 \dagger}$, Ming Gao ${ }^{2,3,4 t}$, Lin Liu ${ }^{1}$, Jinbo Sun ${ }^{1}$, Peng Liu ${ }^{1}$ and \\ Wei Qin ${ }^{1 *}$ \\ ${ }^{1}$ Engineering Research Center of Molecular and Neuro Imaging of Ministry of Education, School of Life Science \\ and Technology, Xidian University, Xi'an, China, ${ }^{2}$ Assisted Reproduction Center, Northwest Women's and Children's \\ Hospital, Xi'an, China, ${ }^{3}$ Department of Urology, Xijing Hospital, Fourth Military Medical University, Xi'an, China, ${ }^{4}$ Department \\ of Andrology, Xiyuan Hospital, China Academy of Chinese Medical Sciences, Beijing, China
}

Recent neuroimaging studies have indicated that abnormalities in brain structure and function may play an important role in the etiology of lifelong premature ejaculation (LPE). LPE patients have exhibited aberrant cortical structure, altered brain network function and abnormal brain activation in response to erotic pictures. However, it remains unclear whether resting-state whole brain functional connectivity (FC) is altered in LPE patients. Machine learning analysis has the advantage of screening the best classification features from high-throughput data (such as FC), which has the potential to identify the pathophysiological targets of disease by establishing classification indicators for patients and healthy controls (HCs). Therefore, the supported vector machine based classification model using FC as features was used in the present study to confirm the most specific FCs that distinguish LPE patients from healthy controls. After feature selection, the remained features were used to build the classification model, with an accuracy $0.85 \pm 0.14$, sensitivity of $0.92 \pm 0.18$, specificity of $0.72 \pm 0.30$, and recall index of $0.85 \pm 0.17$ across 1000 testing groups (100 times 10-folds cross validation). After that, two-sample $t$-tests with family-wise error correction were used to compare these features that occur more than 500 times during training steps between LPE patients and HCs. Four FCs, (1) between left medial part of orbital frontal cortex (mOFC) and right mOFC, (2) between the left rectus and right postcentral gyrus, (3) between the right insula and left pallidum, and (4) between the right middle part of temporal pole and right inferior part of temporal gyrus showed significant group difference. These results demonstrate that resting-state brain FC might be a discriminating feature to distinguish LPE patients from HCs. These classification features, especially the FC between bilateral mOFC, provide underlying abnormal central functional targets in LPE etiology, which offers a novel alternative target for future intervention in LPE treatment.

Keywords: lifelong premature ejaculation, feature selection, functional magnetic resonance imaging, support vector machine, functional connectivity 


\section{INTRODUCTION}

In recent years, more and more neuroimaging studies have found that the etiology of sexual function dysfunction may be related to brain abnormalities, including brain structure, and functional aberrance (Zhao et al., 2015a,b; Chen et al., 2017; Jin et al., 2017; Li et al., 2018). Lifelong premature ejaculation (LPE) is one of the most common male sexual dysfunction diseases. According to the International Society for Sexual Medicine, LPE is defined as "a male sexual dysfunction characterized by ejaculation which always or nearly always occurs prior to or within about $1 \mathrm{~min}$ of vaginal penetration since the first sexual experience; and inability to delay ejaculation on all or nearly all vaginal penetrations; and negative personal consequences, such as distress, bother, frustration and or the avoidance of sexual intimacy (Althof et al., 2014)." Although selective serotonin reuptake inhibitors have been found to produce a side effect of delayed ejaculation in the treatment of depression and have gradually become the first-line drug for clinical treatment of premature ejaculation (PE) (Giuliano and Clement, 2012), the pathophysiological mechanisms of LPE remain unclear. As early as 10 years ago, neuroimaging studies demonstrated that the brain is involved in ejaculation behavior (Holstege et al., 2003; Georgiadis et al., 2007); however, evidence regarding to the role of the brain in the etiology of LPE remains limited, especially at the supraspinal level.

In Zhang et al. (2017), the first neuroimaging study of brain changes in LPE patients was conducted. Subsequently, there have been a few studies reporting brain structural and functional abnormalities in LPE, including by our group. These studies have shown that LPE patients have increased cortical thickness and possible improved sensory ascending conduction efficiency (Guo et al., 2017; Gao et al., 2018), and abnormal brain function either in resting state or during erotic picture stimulation (Zhang et al., 2017; Lu et al., 2018; Yang et al., 2018), which have provided new evidence for the neurobiological etiology of LPE. Recently, machine learning methods have also been used in the analysis of high-throughput brain imaging data to obtain more disease-specific imaging features. For example, classifiers based on brain structure or brain function features have been used to distinguish psychiatric patients from healthy people, to distinguish different subtypes of patients, and to predict remission and non-remission when evaluating therapeutic effects (Fu et al., 2008; Grotegerd et al., 2014; Redlich et al., 2016; Du et al., 2018). These classification features offer useful insight for detecting the biological mechanisms of diseases. Interestingly, a recent study investigating the brain mechanism of venous erectile dysfunction used machine learning classification to distinguish patients from healthy controls, and revealed more various white matter-derived indices that might underlie imaging targets related to the neurobiological etiology of venous erectile dysfunction (Li et al., 2018).

Therefore, in the present study, we aimed to use a machine learning method to classify LPE patients from healthy subjects based on high-throughput resting brain functional connectivity (FC) data, in effort to find the most specific discriminating indicators between LPE patients and healthy controls. We believe our results provide novel information for understanding the neurobiological mechanism of LPE.

\section{MATERIALS AND METHODS}

\section{Participants}

Sixty male adults non-medicated PE patients and sixty male non-drug-using healthy controls (HCs) were recruited in our study. LPE was diagnosed according to the International Society for Sexual Medicine's guidelines for the diagnosis and treatment of premature ejaculation (Althof et al., 2010). All participants underwent history taking and physical examination. Each patient had an intravaginal ejaculatory latency time (IELT) within $1 \mathrm{~min}$. The premature ejaculation diagnostic tool (PEDT) score of each LPE patient was $>11$, but $<5$ for each control. The International Index of Erectile Function score was no less than 21 for all subjects. Participants were excluded if they met any of the following criteria: (1) had a history of alcohol or drug abuse, (2) had a history of psychiatric or neurologic diseases, (3) having a history of head trauma, and (4) had any contra-indication to MRI scanning.

According to the selection standards above, $45 \mathrm{PE}$ patients and 40 HCs were included in the current study. Written informed consent was obtained from all study participants. Research procedures were approved by the ethical committee of the Northwest Women's and Children's Hospital in China, and were conducted in accordance with the Code of Ethics of the World Medical Association (Declaration of Helsinki).

\section{Imaging Data Acquisition}

All subjects underwent a series of image scanning using a 3T GE MR750 scanner at the Department of Radiology, Xijing Hospital, the Fourth Military Medical University, Xi'an, China. A standard 8-channel head coil was used together with a restraining foam pad to minimize head motion and diminish scanner noise. Restingstate functional images were acquired with a single-shot gradient recalled echo planar imaging sequence. (TR/TE: $2000 \mathrm{~ms} / 30 \mathrm{~ms}$, field of view: $240 \times 240 \mathrm{~mm}^{2}$, matrix size: $64 \times 64$, flip angle: $90^{\circ}$, in-plane resolution: $3.75 \times 3.75 \mathrm{~mm}^{2}$, slice thickness: $3.5 \mathrm{~mm}$ with no gaps, 45 axial slices). For each subject, a total of 210 volumes were acquired. High resolution T1-weighted images were collected with a volumetric three-dimensional spoiled gradient recall sequence (TR/TE: $8.2 \mathrm{~ms} / 3.18 \mathrm{~ms}$, field of view: $256 \times 256 \mathrm{~mm}^{2}$, matrix size: $512 \times 512$, flip angle $=9^{\circ}$, in-plane resolution: $0.5 \times 0.5 \mathrm{~mm}^{2}$, slice thickness $=1 \mathrm{~mm}, 196$ sagittal slices). During the resting scanning, subjects were instructed to keep their eyes open and to not think about anything.

\section{Imaging Data Preprocessing}

Functional image preprocessing was carried out using CONN software ${ }^{1}$. Briefly, after excluding the first five images to ensure the signal had reached equilibrium, functional images were corrected for head motion and temporal differences. A participant was excluded if any translation or rotation parameters in subject's data set exceeded $\pm 1 \mathrm{~mm}$ or $\pm 1^{\circ}$,

\footnotetext{
${ }^{1}$ http://web.mit.edu/swg/software.htm
} 
respectively. After this step, 39 patients and $30 \mathrm{HCs}$ remained. After that, outlier detection was performed. Next, the corrected functional images were coregistered to each subject's T1 image without reslicing the image. After that, T1 images were normalized to the Montreal Neurological Institute (MNI) space, which generated a transformed matrix from native space to MNI space. Functional images were then transformed to the MNI space using this matrix and resampled at $2 \times 2 \times 2 \mathrm{~mm}^{3}$. Finally, all images were smoothed with a $6-\mathrm{mm}$ full width at half maximum Gaussian kernel.

To remove spurious sources of variance, time series of each brain voxel were performed by the following steps: (1) linear detrending; (2) regressing out the six head motion parameters and their first-level derivative, the averaged cerebrospinal fluid and white matter signals, and the scrubbing signal from the time series generated by the functional outlier detection (ARTbased identification of scans for scrubbing) process in CONN; (3) $0.01-0.1 \mathrm{~Hz}$ band-pass filtering.

After data preprocessing, time series of each region of interest (ROI) were extracted as the average time series across all voxels in that ROI based on the Anatomical Automatic Labeling (AAL) cortical and subcortical atlas (Tzourio-Mazoyer et al., 2002). In this step, 90 ROI time series were extracted. Finally, the FC coefficient (e.g., Pearson's correlation coefficient) between each pair of these 90 time series was calculated, which resulted in 4005 edges for each subject for subsequent analysis.

\section{Features Selection and Classification Model}

Ten-folds cross validation (CV) was used to assess the reliability of the classification model. Briefly, 69 subjects were randomly separated into 10 groups. Each time, one group in turn was used as a testing group and the other nine groups were used as training group.

Firstly, two sample $T$-test was used as the first step to preliminarily select features from the 4005 edges in training group. The edges with a $p$-value less than $P_{0}$ were selected as initial features. After that, we used a 10 -folds CV based Least Absolute Shrinkage and Selection Operator (CV-LASSO) method to further select features. Briefly, subjects in training group were again randomly separated into 10 groups. Each time, one group in turn was excluded from the dataset, and the LASSO (Sauerbrei et al., 2007) method with mean of square error (MSE) as the cost function was used on the remaining nine groups to narrow down the initial features into the most important features according to the MSE+1SE criteria (Sauerbrei et al., 2007). This step was repeated 10 times, which resulted in 10 different groups of selected features. Finally, the edges that were included in the selected feature group at least $N$ times (i.e., occurring $N$ times) were selected as LASSO features for further analysis. Next, the linear supported vector machine (LSVM) method was used to construct the classification model based on LASSO features in training group, which was implemented using libsvm software ${ }^{2}$. The accuracy, sensitivity, specificity and recall indices of the constructed model were calculated using testing group.

All these steps above were repeated 10 times. As for the setting of $P_{0}, N$, and the cost parameter $c$ in libsvm, we used gridsearch method to find them. These parameters were set at a group of specific values when the accuracy index of the constructed classification model achieved the maximum. The $P_{0}$ was set from 0.025 to 0.2 with a step of 0.025 and including $0.001,0.005$, and 0.01 . The $N$ was set from 1 to 10 with a step of 1 . The $c$ was set from 0.1 to 2 with a step of 0.1 .

To avoid the random group effect, we repeated the 10 -folds CV 100 times. For each time, a new random group was split. The mean \pm standard deviation of each index across the 1000 testing groups $(10 \times 100)$ was used to assess the performance and stability of the constructed model. Finally, 1000 times permutation test (group label permutation) was performed to check if our results were significantly different from random label. Figure 1 illustrates the framework of our study.

${ }^{2}$ https://www.csie.ntu.edu.tw/ cjlin/libsvm/

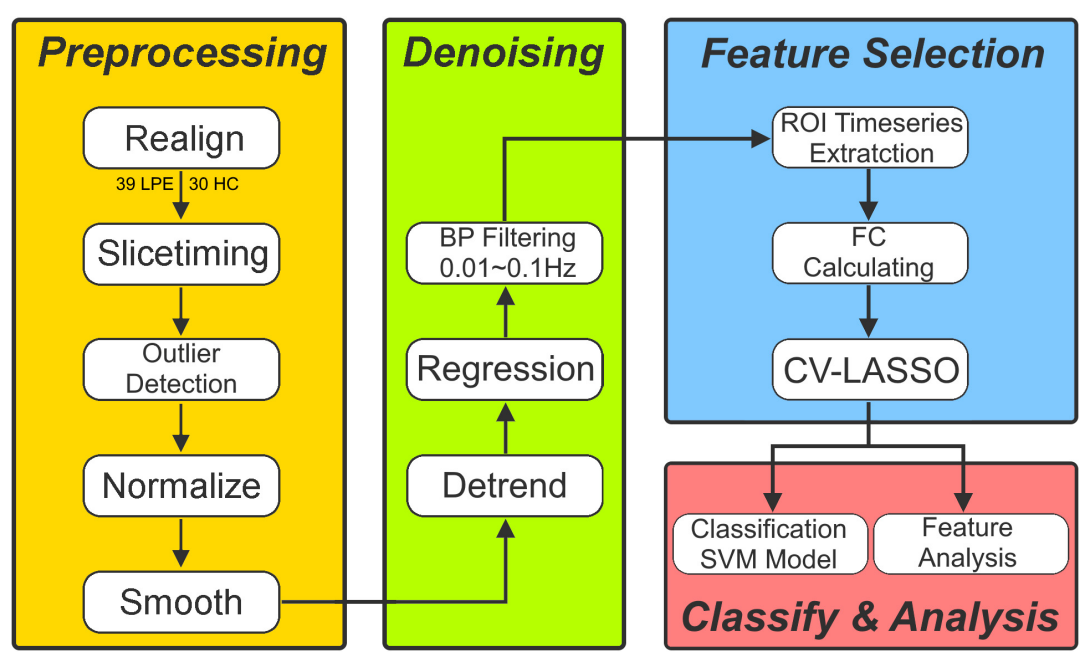

FIGURE 1 | The framework of study procedure. 


\section{RESULTS}

\section{Clinical and Demographic Characteristics}

Clinical and demographic characteristics of the subjects are shown in Table 1. The PEDT scores of LPE patients were significantly higher than those of HCs, and the IELT of LPE patients was dramatically shorter than that of HCs.

\section{Classification}

The 100 times 10 -fold CV results of the model were shown in Table 2. The accuracy, sensitivity, specificity and recall indices of the classification model were $0.8490 \pm 0.1401,0.9238 \pm 0.1817$, $0.7250 \pm 0.3038$, and $0.8506 \pm 0.1740$, respectively. Figure 2B displays the receiver operating characteristic curve (ROC) of the classification model, and the AUC was 0.8047 . Figure 3 shows the permutation test results of our constructed classification model. Together, these results demonstrate the stability of our classification model and the reliability of our method.

After counting the occurring time of each LASSO feature in 100 times 10 -fold CV, 5 LASSO features with occurring time larger than 500 were finally selected as the most important features in classification, which involved brain regions in the frontal, temporal and limbic lobes (Table 3), since we believed that features which occurring time less than 500 were to a large extent relied on the specific splitting group. Figure 2A gives the spatial distribution of these 5 LASSO features.

\section{DISCUSSION}

By using a machine learning classification method to assess resting-state brain function in LPE patients, the present study screened 9.042 (average across every training step during 100 times 10-folds CV) out of 4005 FC features to construct the optimal classifier, which could separate patients from healthy people with an accuracy of 0.85 . These FC features are mainly

TABLE 1 | Clinical and demographic characteristics.

\begin{tabular}{lccc}
\hline & HC $(\boldsymbol{n}=\mathbf{3 0})$ & LPE $(\boldsymbol{n}=\mathbf{3 9})$ & $\boldsymbol{P}$-value \\
\hline Age (years) & $31.33 \pm 2.77$ & $30.52 \pm 5.06$ & 0.44 \\
PEDT score & $0.80 \pm 1.40$ & $17.50 \pm 1.96$ & $<0.0001$ \\
IIEF-5 score & $24.5 \pm 0.63$ & $24.29 \pm 0.47$ & 0.17 \\
IELT (min) & $644.00 \pm 366.47$ & $37.02 \pm 16.75$ & $<0.0001$
\end{tabular}

Data were presented as mean $\pm S D$. HC, healthy control; IELT, intravaginal ejaculatory latency time; IIEF-5, International Index of Erectile Function-5; LPE, lifelong premature ejaculation; PEDT, Premature ejaculation diagnostic tool. distributed in some areas in the frontal, temporal, and parietal cortex, and limbic system. Compared with previous studies, our results provide more novel FC-derived indicators through a strategy of classification research to understand the potential abnormalities of brain function in LPE patients.

The classification algorithm in machine learning is useful for exploring the best classification features from high-throughput information, in which multivariate decoding algorithms like supported vector machine are trained on a portion of the data by weighting all connections in order to separate the known clinical status from HCs, rather than testing each connection independently for group differences. The whole brain functional connections belong to a high-throughput data set, in which there are more than 4000 FCs in the whole brain when the human brain is divided into 90 ROIs. In our present study, through CV-LASSO dimension reduction method, we have obtained a classifier with a relatively high accuracy to individually distinguish LPE patients from HCs. This machine learning-based classification approach based on resting-state FC has previously been used to distinguish patients with brain disorders from $\mathrm{HCs}$, and responders from non-responders in clinical drug or invention trials (van Waarde et al., 2015; Sarpal et al., 2016; Arbabshirani et al., 2017; Plaschke et al., 2017). Therefore, the features based on the resting-state FC in our present study may be biomarkers that allow the classification of individual LPE patients.

Among the five selected features which occur more than 500 times in training step during 100 times 10 -fold CV, the connections between bilateral mOFC had the highest weight according to our results. OFC has been implicated in ejaculation control. A previous positron emission tomography study has reported a remarkable decrease of regional cerebral blood flow throughout the prefrontal cortex during ejaculation in male volunteers (Holstege et al., 2003; Georgiadis et al., 2007). Our previous study has also found abnormal prefrontal control function in LPE patients by using classical inhibitory control tasks, and reduced FC between the inferior frontal cortex and the frontal pole was found in LPE patients (Yang et al., 2018). Together with the present results that the synchronized activity of the mirror symmetric OFC had absolute superiority in discriminating LPE patients from the healthy controls, it further indicates that the OFC is likely closely involved in the etiology of LPE, and the OFC-related inhibitory control function may be impaired in LPE patients, which might cause the loss of the inhibitory tone on ejaculation in LPE patients.

Besides, most of the other FC related regions in the current study were also reported to be related to male sexual physiology. Zhang et al. (2017) have found that the insula and middle part of temporal gyrus showed abnormal activation in response to erotic stimulation, and also had aberrant regional activity

TABLE 2 | Performance information of classification model.

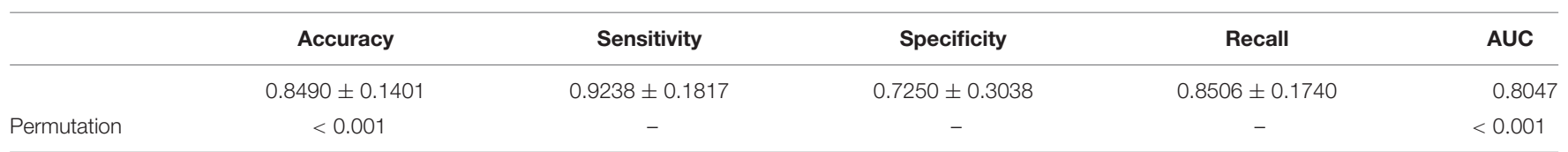

AUC, area under the curve; Permutation, 1000 Permutation test. 


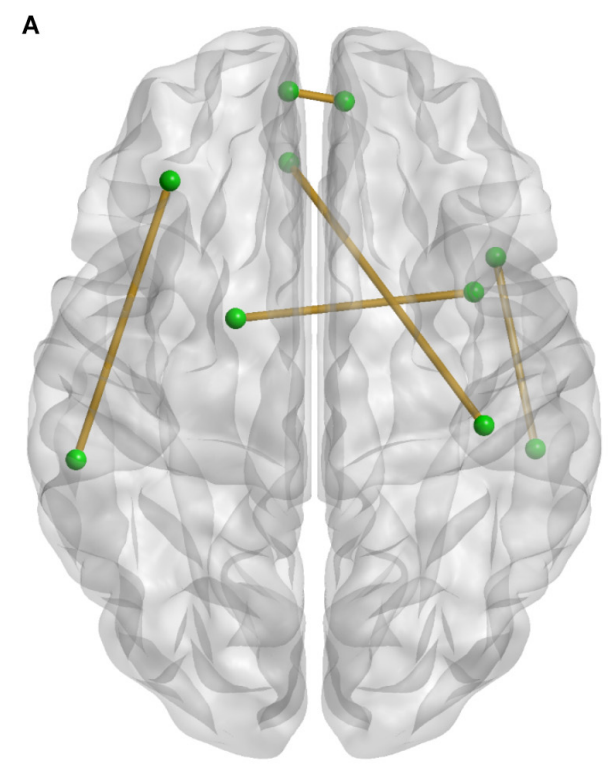

B

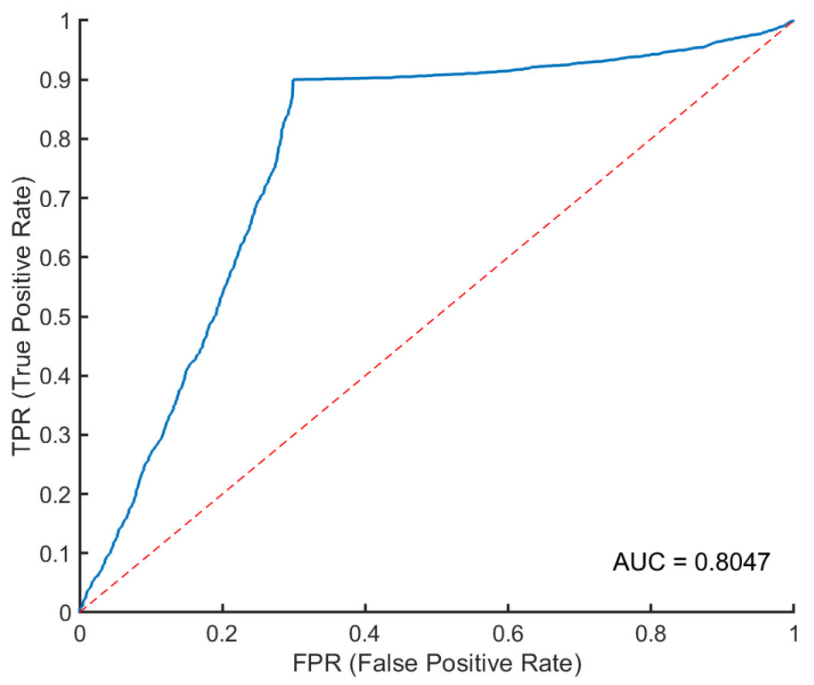

FIGURE 2 | (A) The spatial distribution of five selected LASSO features and (B) the receiver operating characteristic (ROC) curve of the classification model. LASSO, least absolute shrinkage and selection operator.
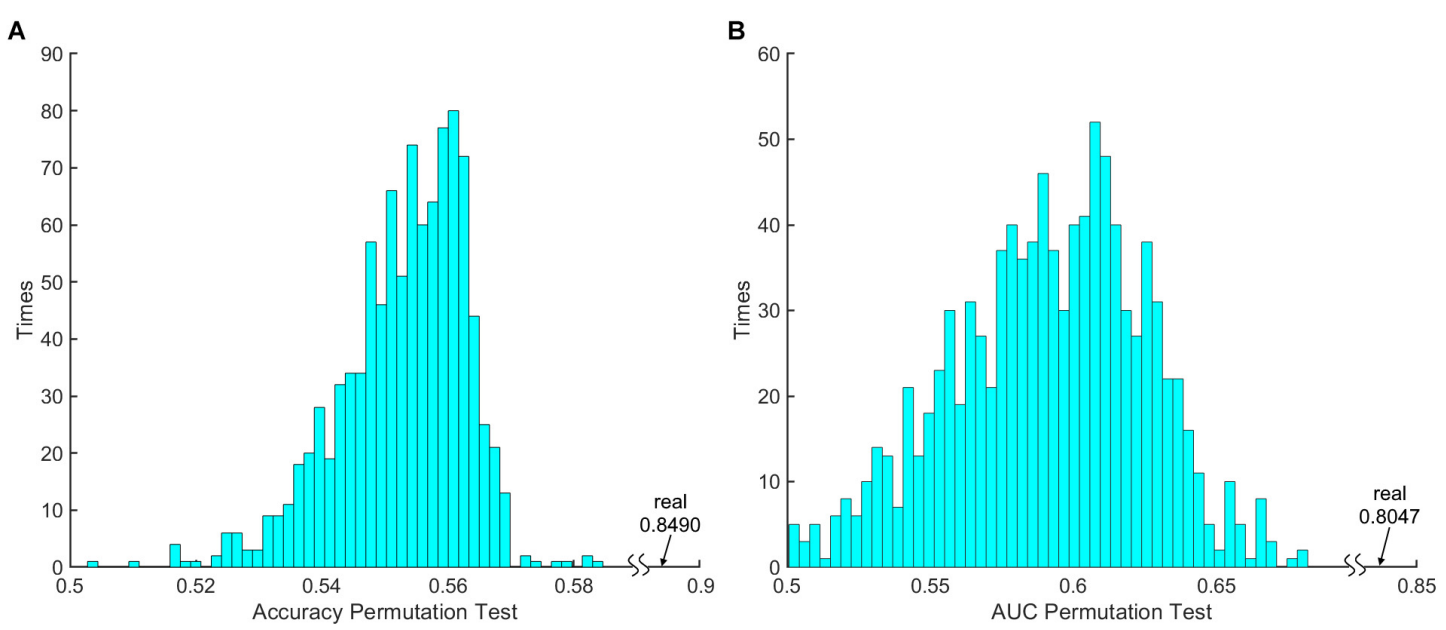

FIGURE 3 | The 1000 times permutation test results of (A) classification model accuracy index and (B) area under curve (AUC).

TABLE 3 | Detailed information of five selected LASSO features.

\begin{tabular}{|c|c|c|}
\hline \multicolumn{2}{|c|}{ Edge } & \multirow[t]{2}{*}{ Weight } \\
\hline $\mathrm{HC}>\mathrm{PE}$ & & \\
\hline Frontal_Med_Orb_L & Frontal_Med_Orb_R & 0.4874 \\
\hline Rectus_L & Postcentral_R & 0.0020 \\
\hline Insula_R & Pallidum_L & 0.1270 \\
\hline \multicolumn{3}{|l|}{$\mathrm{HC}<\mathrm{PE}$} \\
\hline Frontal_Mid_L & SupraMarginal_L & 0.1370 \\
\hline Temporal_Pole_Mid_R & Temporal_Inf_R & 0.2466 \\
\hline
\end{tabular}

and FC during resting state in LPE patients. By using cerebral cortical thickness measurements, we once reported widespread cortical thickening in the orbitofrontal, middle frontal, and supramarginal gyrus in LPE patients (Guo et al., 2017). A recent fMRI study detected the resting-state FC density in LPE patients, which found that anterior cingulate cortex, insula, and precuneus had increased long-range FC density in LPE patients compared to healthy controls (Lu et al., 2018). Although the role of gyrus rectus and postcentral gyrus in LPE has not been reported yet, the gyrus rectus is located in the medial orbital gyrus and plays an inhibition role in sexual arousal (Stoleru et al., 2012), and sensory stimuli from penis could induce Rolandic opercula area and postcentral gyrus activation (Stoleru et al., 2012). 
So, despite our results were derived from a data-driven method, these classification features that are involved in ejaculation and other sexual behaviors extend our knowledge of the central pathophysiology in LPE patients.

There are several limitations in the current study. We only included LPE patients without secondary PE patients. So, we do not know if our classifier was specific to LPE or trans-disease subtypes across all PE patients. Further research is necessary to include more subtypes of PE patients for classification studies. In addition, other than FC, brain gray matter and white matter structure have often been used as classification indicators. $\mathrm{Li}$ et al. (2018) have used white matter indicators to successfully distinguish venous erectile dysfunction patients from HCs. These measures were not included in the present study, but multimodal brain imaging information should be used in future classification studies of PE.

\section{CONCLUSION}

By using machine learning analysis, this study identified potential neuroimaging markers based on resting-state whole brain FC that could distinguish LPE patients from HCs. These classification features provide novel information for explaining the central mechanisms of LPE, and further emphasize the potential functional abnormalities of the central inhibitory control network and sexual-related regions in LPE patients.

\section{ETHICS STATEMENT}

Written informed consent was obtained from all study participants. Research procedures were approved by the ethical

\section{REFERENCES}

Althof, S. E., Abdo, C. H., Dean, J., Hackett, G., McCabe, M., McMahon, C. G., et al. (2010). International society for sexual medicine's guidelines for the diagnosis and treatment of premature ejaculation. J. Sex. Med. 7, 2947-2969. doi: 10.1111/j.1743-6109.2010.01975.x

Althof, S. E., McMahon, C. G., Waldinger, M. D., Serefoglu, E. C., Shindel, A. W., Adaikan, P. G., et al. (2014). An update of the international society of sexual medicine's guidelines for the diagnosis and treatment of premature ejaculation (PE). J. Sex. Med. 11, 1392-1422. doi: 10.1111/jsm.12504

Arbabshirani, M. R., Plis, S., Sui, J., and Calhoun, V. D. (2017). Single subject prediction of brain disorders in neuroimaging: promises and pitfalls. Neuroimage 145(Pt B), 137-165. doi: 10.1016/j.neuroimage.2016.02.079

Chen, J., Chen, Y., Chen, G., Dai, Y., Yao, Z., and Lu, Q. (2017). Altered brain networks in psychogenic erectile dysfunction: a resting-state fMRI study. Andrology 5, 1073-1081. doi: 10.1111/andr.12411

Du, Y., Fu, Z., and Calhoun, V. D. (2018). Classification and prediction of brain disorders using functional connectivity: promising but challenging. Front. Neurosci. 12:525. doi: 10.3389/fnins.2018.00525

Fu, C. H., Mourao-Miranda, J., Costafreda, S. G., Khanna, A., Marquand, A. F., Williams, S. C., et al. (2008). Pattern classification of sad facial processing: toward the development of neurobiological markers in depression. Biol. Psychiatry 63, 656-662. doi: 10.1016/j.biopsych.2007.08.020

Gao, M., Yang, X., Liu, L., Fei, N., Xi, Y., Guo, F., et al. (2018). Abnormal white matter microstructure in lifelong premature ejaculation patients identified by committee of the Northwest Women's and Children's Hospital in China, and were conducted in accordance with the Code of Ethics of the World Medical Association (Declaration of Helsinki).

\section{AUTHOR CONTRIBUTIONS}

JS, PL, XY, and WQ contributed to the conception and design. LL contributed to the acquisition of data. ZX and XY contributed to the data analysis and manuscript writing. XY, LL, and ZX contributed to the interpretation of the results.

\section{FUNDING}

This study was financially supported by National Basic Research Program of China under grant nos. 2015CB856403 and 2014CB543203, the Science and Technology Projects of Xi'an, China under grant no. 201809170CX11JC12, the National Natural Science Foundation of China under grant nos. 81471811 and 81471738, the Development Funds of Shaanxi Science and Technology Agency of China under grant no. 2018SF/091, Shaanxi Health and Family Planning Commission Foundation of China for Youths under grant no. 2016E04, and Xi'an Science and Technology Development Funds of China under grant no. 2016052SF/YX08.

\section{ACKNOWLEDGMENTS}

We thank Lesley McCollum, Ph.D., from Liwen Bianji, Edanz Editing China (www.liwenbianji.cn/ac), for editing the English text of a draft of this manuscript.

tract-based spatial statistical analysis. J. Sex. Med. 15, 1272-1279. doi: 10.1016/ j.jsxm.2018.07.012

Georgiadis, J. R., Reinders, A. A., Van der Graaf, F. H., Paans, A. M., and Kortekaas, R. (2007). Brain activation during human male ejaculation revisited. Neuroreport 18, 553-557. doi: 10.1097/wnr.0b013e3280b10bfe

Giuliano, F., and Clement, P. (2012). Pharmacology for the treatment of premature ejaculation. Pharmacol. Rev. 64, 621-644. doi: 10.1124/pr.111.004952

Grotegerd, D., Stuhrmann, A., Kugel, H., Schmidt, S., Redlich, R., Zwanzger, P., et al. (2014). Amygdala excitability to subliminally presented emotional faces distinguishes unipolar and bipolar depression: an fMRI and pattern classification study. Hum. Brain Mapp. 35, 2995-3007. doi: 10.1002/hbm.22380

Guo, F., Xi, Y. B., Gao, M., Liu, L., Fei, N. B., Qin, W., et al. (2017). Alterations in cortical thickness in nonmedicated premature ejaculation patients: a morphometric MRI study. J. Magn. Reson. Imaging 47, 656-662. doi: 10.1002/ jmri.25808

Holstege, G., Georgiadis, J. R., Paans, A. M., Meiners, L. C., van der Graaf, F. H., and Reinders, A. A. (2003). Brain activation during human male ejaculation. J. Neurosci. 23, 9185-9193. doi: 10.1523/jneurosci.23-27-09185.2003

Jin, C., Guan, M., Dong, M., Wu, J., He, Z., Chen, X., et al. (2017). Aberrant baseline brain activity in psychogenic erectile dysfunction patients: a resting state fMRI study. Brain Imaging Behav. 12, 1393-1404. doi: 10.1007/s11682-017-9805-9

Li, L., Fan, W., Li, J., Li, Q., Wang, J., Fan, Y., et al. (2018). Abnormal brain structure as a potential biomarker for venous erectile dysfunction: evidence from multimodal MRI and machine learning. Eur. Radiol. 28, 3789-3800. doi: 10.1007/s00330-018-5365-7 
Lu, J., Zhang, X., Wang, H., Qing, Z., Han, P., Li, M., et al. (2018). Short- and longrange synergism disorders in lifelong premature ejaculation evaluated using the functional connectivity density and network property. Neuroimage Clin. 19, 607-615. doi: 10.1016/j.nicl.2018.05.025

Plaschke, R. N., Cieslik, E. C., Muller, V. I., Hoffstaedter, F., Plachti, A., Varikuti, D. P., et al. (2017). On the integrity of functional brain networks in schizophrenia, Parkinson's disease, and advanced age: evidence from connectivity-based single-subject classification. Hum. Brain Mapp. 38, 58455858. doi: 10.1002/hbm.23763

Redlich, R., Opel, N., Grotegerd, D., Dohm, K., Zaremba, D., Burger, C., et al. (2016). Prediction of individual response to electroconvulsive therapy via machine learning on structural magnetic resonance imaging data. JAMA Psychiatry 73, 557-564. doi: 10.1001/jamapsychiatry.2016.0316

Sarpal, D. K., Argyelan, M., Robinson, D. G., Szeszko, P. R., Karlsgodt, K. H., John, M., et al. (2016). Baseline striatal functional connectivity as a predictor of response to antipsychotic drug treatment. Am. J. Psychiatry 173, 69-77. doi: 10.1176/appi.ajp.2015.14121571

Sauerbrei, W., Royston, P., and Binder, H. (2007). Selection of important variables and determination of functional form for continuous predictors in multivariable model building. Stat. Med. 26, 5512-5528. doi: 10.1002/sim.3148

Stoleru, S., Fonteille, V., Cornelis, C., Joyal, C., and Moulier, V. (2012). Functional neuroimaging studies of sexual arousal and orgasm in healthy men and women: a review and meta-analysis. Neurosci. Biobehav. Rev. 36, 1481-1509. doi: 10. 1016/j.neubiorev.2012.03.006

Tzourio-Mazoyer, N., Landeau, B., Papathanassiou, D., Crivello, F., Etard, O., Delcroix, N., et al. (2002). Automated anatomical labeling of activations in SPM using a macroscopic anatomical parcellation of the MNI MRI single-subject brain. Neuroimage 15, 273-289. doi: 10.1006/nimg.2001. 0978 van Waarde, J. A., Scholte, H. S., van Oudheusden, L. J., Verwey, B., Denys, D., and van Wingen, G. A. (2015). A functional MRI marker may predict the outcome of electroconvulsive therapy in severe and treatment-resistant depression. Mol. Psychiatry 20, 609-614. doi: 10.1038/mp.2014.78

Yang, X., Gao, M., Zhang, L., Liu, L., Liu, P., Sun, J., et al. (2018). Central neural correlates during inhibitory control in lifelong premature ejaculation patients. Front. Hum. Neurosci. 12:206. doi: 10.3389/fnhum.2018.00206

Zhang, B., Lu, J., Xia, J., Wang, F., Li, W., Chen, F., et al. (2017). Functional insights into aberrant brain responses and integration in patients with lifelong premature ejaculation. Sci. Rep. 7:460. doi: 10.1038/s41598-017-00421-3

Zhao, L., Guan, M., Zhang, X., Karama, S., Khundrakpam, B., Wang, M., et al. (2015a). Structural insights into aberrant cortical morphometry and network organization in psychogenic erectile dysfunction. Hum. Brain Mapp. 36, 44694482. doi: 10.1002/hbm.22925

Zhao, L., Guan, M., Zhu, X., Karama, S., Khundrakpam, B., Wang, M., et al. (2015b). Aberrant topological patterns of structural cortical networks in psychogenic erectile dysfunction. Front. Hum. Neurosci. 9:675. doi: 10.3389/ fnhum.2015.00675

Conflict of Interest Statement: The authors declare that the research was conducted in the absence of any commercial or financial relationships that could be construed as a potential conflict of interest.

Copyright (C) 2019 Xu, Yang, Gao, Liu, Sun, Liu and Qin. This is an open-access article distributed under the terms of the Creative Commons Attribution License (CC BY). The use, distribution or reproduction in other forums is permitted, provided the original author(s) and the copyright owner(s) are credited and that the original publication in this journal is cited, in accordance with accepted academic practice. No use, distribution or reproduction is permitted which does not comply with these terms. 